\title{
CUENCA UNA SOCIEDAD INTERCULTURAL. LOS DESAFÍOS DE LA ACADEMIA Y LA CIUDAD
}

\author{
CUENCA AN INTERCULTURAL SOCIETY. THE CHALLENGES \\ OF THE ACADEMY AND THE CITY
}

Fanny Tubay Zambrano'

fannytubay@hotmail.com
Fecha de recepción: 02 de marzo de 2018

Fecha de aceptación: 20 de junio 2018.

Código JEL: Z1

Código DOI: 10.25097/rep.n28.2018.05

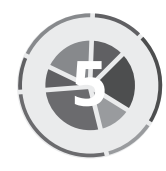

\section{Resumen}

Las transformaciones sociales en Cuenca, han dado paso a la constitución de diversas formas de ser y ejercer ciudadanía, en un contexto que se ha visto alterado por movimientos migratorios, que reconstruyen y cambian la dinámica de espacios ciudadanos y perspectivas poblaciones. El estudio muestra que si bien, la ciudad se reconoce multicultural, requiere una praxis intercultural, para mediar, superar conflictos y mejorar condiciones de vida. Por tanto, se plantea a la academia y a la ciudad, como espacios macros, para potenciar la interacción en sociedades interculturales, bajo una visión integradora que intersecta ambos imaginarios. Se aplica una metodología cualitativa, con herramientas auto etnográficas y entrevistas interactivas, así como de una investigación documental. Los resultados dan cuenta que los dispositivos de ciudad y academia, permiten un acercamiento oportuno cuando las relaciones se flexibilizan, y se toman los espacios como escenario de aprendizaje y práctica para el enriquecimiento social.

Palabras Claves: Cuenca, sociedades interculturales, multicultural; ciudad; academia.

\begin{abstract}
The social transformations in Cuenca has given way to the constitution of diverse forms of being and exercise of citizenship, in a context that has been altered by migratory movements, which reconstruct and change the dynamics of citizens' spaces and perspectives. The study shows that although the city is recognized as multicultural, it requires an intercultural practice to mediate, overcome conflicts and improve living conditions. Therefore, the academic and city are proposed, as macro spaces, to enhance interaction in intercultural societies, under an integrating vision that intersects both imaginaries. A qualitative methodology is applied, with automatic tools and interactive interviews, as well as a documentary investigation. The results of the account and the devices of the city and the academy, allow a timely approach when relationships are relaxed, and spaces are taken as a learning and practice scenario for social enrichment.
\end{abstract}

Keywords: Cuenca, intercultural societies, multicultural, city, academy. 


\section{Introducción}

En los últimos años se ha evidenciado la necesidad política de introducir definiciones como multiculturalismo e interculturalidad, tanto en las agendas políticas de las naciones como en los imaginarios sociales, con el propósito de enunciar la mirada heterogénea de la diversidad cultural, desde un ámbito discursivo y en medio de álgidos movimientos migratorios. Al mismo tiempo, se han acentuado las brechas, producto de esos flujos poblacionales, motivados por las guerras, las crisis económicas, políticas o sociales, o por temas ambientales. Esos cambios acelerados, han hecho que además de poner el "mundo en movimiento" también se ponga en movimiento estas definiciones y se preste (...) más atención a los límites, los intersticios, los lugares y -especialmente- los "no-lugares" del mundo global" (Eckholt, 2018, p.120), como espacios de atención emergente, dada la complejidad de su entramado socio cultural. En consecuencia, esa serie de conflictos de convivencia, propician en las ciudades y sociedades de acogida, a modo de resistencia, aquellas "comunitas que emergen donde la estructura social no lo hace". (Turner, 1969, p. 371).

Es por eso, que este trabajo reúne una revisión de enfoques teóricos que se intersectan y abordan también la "ssociedad del conocimiento", la "nueva economía" y la "economía basada en el conocimiento" (Bianco, Lugones, Peirano y Salazar, 2016, p.4), como puntos clave, para comprender el camino que debe transitar una sociedad, cada vez menos quieta y más cercana a la "hibridación cultural" Canclini (2012).

En medio de esa hibridación que da paso también a nuevas formas de vida, la ciudad de Cuenca se proyecta en medio de un escenario natural y cultural, desdibujado como el "ideal de la urbe que ofrece un espacio de paz y libertad tras sus muros para quienes la habitan" (Eckholt, 2018, p.117) constituyéndose en un nexo que, pone en valor la dinámica poblacional, en un sentido multi e intercultural.

Finalmente, la investigación aterriza en el campo de las ciencias sociales, basándose en teorías que fluctúan desde el enfoque intercultural y de la praxis de un conjunto de ciudadanos, que por medio de la etnografía revelan "nuevos comportamientos individuales, relaciones sociales, elementos materiales y otras cosas o hechos sociales" (Schensul y LeCompte, 1999, p.114) que ponen en perspectiva un modelo de ciudad, que brinda las condiciones propicias para asentarse y confluir globalmente.

\section{Estado del arte}

Es innegable que, en la actualidad lo diverso se sigue considerando un conflicto más que una oportunidad, sobre todo cuando la diferencia remarca las asimetrías categóricas, que no permiten percibir que "en la medida en que ponemos menos el acento sobre la forma, la cultura, y más sobre el sujeto que actúa y por tanto que interactúa". (Odina y Del Olmo, 2009, p.17) adquirimos una amplia consciencia colectiva, condicionada en gran medida por las estructuras sociales que promueven los estados.

En ese contexto, es importante iniciar una revisión desde el accionar del estado nación ecuatoriano, con respecto a los avances sociales que "cuestionan las promesas falaces y racistas de un "crisol cultural" supuestamente "neutral ante diferencias étnico-raciales" de la sociedad hegemónica" (Dietz, 2017, p.197) autoproclamados multi e interculturales. Si bien, este ha hecho eco de una retórica que incide en los objetivos para alcanzar mejores y armónicas relaciones de convivencia, enfocadas en el buen vivir, como filosofía y política de estado. A través de instrumentos legislativos como la Ley de Educación Intercultural (2011), la Constitución de la República del Ecuador (2008) y el Plan Nacional del Buen Vivir (2009), que han servido como modelos garantistas de los deberes y derechos tanto de ciudadanos como de la naturaleza. Sin embargo, como ya lo apuntábamos, estos intentos más que revocar en resultados positivos, han colocado y visibilizado sobre la palestra, una serie de tensiones, que a falta de mecanismos que 
introduzcan estas nuevas políticas en la sociedad, irrumpen las posibles relaciones y acuerdos ciudadanos. Es por ello, que resulta emergente reflexionar el momento actual y dar paso al reconocimiento de los puntos de inmovilización de la estructura gubernamental y su legislación. Cuyos esfuerzos por tener una reconocida legislación en el mundo, no han sido suficientes, pues es ya casa adentro que se puede "sentí-pensar" (Galeano, 2014) ese momento pausado, de un discurso que no llega a articular ciudadanía, cultura y naturaleza, como medios y fines para lograr constituirse en las tan proclamadas "intercultural cities", Zapata (2015) o ciudades interculturales.

Dicha semántica narrativa, trasciende a un plano social a través de una definición del termino intercultural que "aparece en Francia en 1975, en el marco de las acciones sociales y educativas" (Pretceille y Prat, 2001, p.33), y que, en repetidas ocasiones, se lo ha asociado como sinónimo o parte de - multiculturalismo. Siendo así, necesario partir diferenciando dos conceptos claves, para comprender como se asientan y dialogan los conglomerados ciudadanos, en las arenas de esa multi e interculturalidad.

Acercándonos a sus definiciones y a los roles que estos ejercen en las construcciones de representaciones sociales, Rego (2010), define la multiculturalidad, como es espacio donde "coexisten diversas culturas" y a la interculturalidad "como un modelo social al que aspirar, que parte de la diversidad cultural para promover la interrelación, el diálogo y el enriquecimiento". (Rego, 2010, p.88). Esta interconexión mutua, percibe a la multiculturalidad como ese primer paso para iniciarse en una interculturalidad con "aplicaciones muy diversas y múltiples orientaciones". (Pretceille y Prat, 2001, p.33, en un proceso comunicativo "que tiene en la negociación de significados uno de sus más conspicuos referentes práxicos". (Rego, 2010, p.101)

La coexistencia de la pluralidad y diversidad cultural, se acentúan como las grandes líneas en las que transgrede la multiculturalidad hasta un momento puntual, en adelante, luego del establecimiento de esas unificaciones sociales, a modo de "comunitas" y no de "comunidades" diferenciadas, una como "la relación social" y otra como "un área de la vida común" (Turner, 1969 , p.360), es la interculturalidad que se encarga de dinamizar esas maneras de coexistir, en un universo de diferencias donde se construyen puentes y oportunidades para todos.

En esas comunitas, convertidas en sociedades en constante interrelación, donde a pesar que "cada actor social tiene su propia visión de mundo, sus intereses, sus proyectos y sus ideas particulares de cómo llevarlos adelante". (Mato, 2009, p. 33), se consensuan espacios de acercamiento y respeto por el otro, dando cabida a un pensamiento que invita a "cambiar el paradigma con el cual operacionalizamos a una sociedad como homogénea y reconocer la adopción de perspectivas de interacción intercultural y experiencias multiculturales" (Montero, 2006, p.2). incorporando elementos, tales como "la inclusión de la naturaleza en la ciudad" (Cordero, Vanegas y Hermida, 2015, p.108), conjugando naturaleza y sujeto, como secuencias interconectadas.

Es ese ámbito donde indiscutiblemente, tiene un rol fundamental una educación integral, que rompe las barreras de la escuela y se aleja de las concepciones del "fundamentalismo étnico, nacional o religioso". (Coulby, 2006, p.246), entendiendo la diversidad como riqueza, recurso y ganancia. Se puede reconocer que "la producción, difusión y aplicación de conocimiento en sus diversas formas, contribuyen de manera eficaz tanto a la mejora de la competitividad (...) como a la calidad de vida de sus habitantes, convirtiéndose en objetivo prioritario" (Del Valle, 2009, p.118).

Paralelamente, otras lógicas discursivas, dejan ver que "las naciones democráticas en todo el mundo tienen la tarea de gestionar temas educativos complejos cuando están intentando dar respuesta a los problemas que derivan de la emigración internacional" (Frutos, 2015, p.16), de los movimientos migratorios nacionales, de los conflictos por cuestiones de género, origen o bagaje cultural, y que traen consigo los ciudadanos asentados en un determinado territorio. Siendo estas maneras de conflictos, las que permiten poner en debate perspectivas múltiples, de un sistema intercultural integral, que requiere la "transformación de las estructuras, instituciones y relaciones sociales, y la construcción de condiciones de estar, ser, pensar, conocer, aprender, 
sentir y vivir distintas" (Walsh: 2003, p. 4), traspasando las fronteras de la academia y la ciudad, en un espacio de fundición. En base a este panorama, podemos constatar que ya desde la escuela de Frankfurt, se hablaba de transformaciones mundiales en el marco de la sociedad, aseverando que son "todas las áreas de la actividad humana, las que deben mover las estrategias académicas (Marcovitch, 2002, p.18), para consolidar mejores relaciones e interacciones entre entes sociales y no sociales. Ese diálogo abierto, se inscribe "una universidad (...) en el marco de una contrauniversidad, (Marcuse 1968, p. 94) que en los actuales momentos, bajo una "noción moderna de "campus" reclama como objetivos principales, "el retorno de la presencia activa de la naturaleza dentro del tejido urbano y el reencuentro de un nuevo equilibrio entre ambos factores vistos como ingredientes básicos del espacio habitable". (Roca Blanch, 2011, p. 96).

Resulta interesante percibir que estas formas de educación, que transgreden las clases tradicionales y otorgan "el derecho a discutir y a manifestarse no sólo en el aula, sino en todo el territorio de la Universidad". (Marcuse, 1968, p.96), abren el paso para considerar a "la calle como espacio lúdico por excelencia en el medio urbano y en consecuencia, donde se producen las alteraciones" (Hernández, 2004, p.152), que van "construyendo una relación positiva entre las distintas culturas y asumir con responsabilidad los destinos de nuestro futuro común." (Tum, 2002, p.67).

Llegando a convertir ese contexto ciudadano, en el laboratorio para explorar las diferencias, vivir el conflicto, buscar soluciones y hacer partícipes a voces y minorías que "pueden haber quedado al margen del proceso de construcción nacional, principalmente por estrategias excluyentes de la mayoría, pero también, en alguna medida, por estrategias aislacionistas propias" (Enguita, 2001, p.1).

En términos globales, hemos de pasar de ser una academia que consume conocimiento a una que lo produce, de la mano de una ciudadanía participativa constituida por una sociedad plural, que valora la cultura y la naturaleza como ejes fundamentales para su armoniosa convivencia. Evocando la importancia de "hacer que la educación de los alumnos, incluyendo su instrucción académica, considere el capital social como activo de formación efectiva para una sociedad multicultural" (Rego, 2010, p.97), teniendo a la ciudad como escenario clave para su desarrollo.

\section{Metodología}

Este estudio procura comprender, por medio de una metodología mixta, los modos como se construyen las sociedades interculturales, teniendo como bases u objetos de estudio, la ciudad y la academia. Para el fin, se aplican herramientas metodológicas como las entrevistas interactivas y la auto-etnografía, que forman parte de la investigación de enfoque cualitativo; y de un análisis documental-reflexivo, que articula la teoría con aquello que ha sido recogido en el campo, durante diez meses.

Desde la perspectiva cualitativa, se han aplicado entrevistas de tipo interactivas, con el objetivo "que todos los participantes, generalmente de dos a cuatro personas, incluido el investigador principal, actúen como investigadores y como participantes de la investigación" (Berger y Ellis, 2002, p.857), propiciando un diálogo espontáneo que, permite analizar los resultados a partir de ellos, desde un "proceso dialéctico que transciende el significado particular que se ha dado y lo lleva a un significado más global y universal compartido por todos" (Montero, 2006, p.10). Estas entrevistas interactivas, "ofrecen oportunidades para que los investigadores y los entrevistados reflexionen con autoconsciencia" (Berger y Ellis, 2002, p. 852) los procesos de diálogo, a partir de herramientas más próximas a las realidades que se observa. En ese punto, ha sido necesario contar con cinco actores claves, de diversos orígenes, con tiempos de permanencia que van desde uno hasta cuatro años en la ciudad de Cuenca.

A estas personas se les ha entrevistado con un guion de preguntas, bajo un sentido de orientación inicial que marca el punto de partida, pero no es un destino estricto, ya que muchas de las preguntas han sido flexibilizadas o en su defecto, han sido reorientadas durante las 
conversaciones, sin que esto cambie la intención de la investigación, más bien han servido para familiarizarse y generar un ámbito de comodidad y confianza con el entrevistado. Las preguntas iniciales se propusieron de la siguiente manera:

¿Cuáles son los motivos que te trajeron a vivir en Cuenca?

¿Hay sitios de la ciudad que los sientes cómodos o familiares? ¿Cuáles son? y ¿Por qué?

¿Cuáles sitios de la ciudad prefieres para pasear?

Desde la misma mirada cualitativa, también se ha hecho uso de la herramienta auto-etnográfica, con el propósito de conocer la perspectiva del investigador, situándolo "en el centro de la investigación como un autor integrado, que relaciona su reflexión a lo desconocido, y al conocimiento creado por estas relaciones y las evidencias interpretadas" (Montero, 2010, p. 9). Su experiencia es validada como un hecho correlativo, que sirve para comprender la dinámica social a través de "narrativas de estilo autobiográfico e investigativo que conectan lo personal con las experiencias culturales" (Berger y Ellis, 2002, p.859).

Este estudio envuelve a colaboradores, que voluntariamente dan "cabida tanto a los relatos personales y/o autobiográficos como a las experiencias del etnógrafo como investigador - ya sea de manera separada o combinada- situados en un contexto social y cultural. (Blanco, 2012 , p. 55). De la misma manera, estas personas han autorizado el uso de sus nombres y datos requeridos para el análisis de los resultados. No obstante, se ha optado por codificar sus entrevistas y auto-etnografía, para facilitar la lectura en el texto, haciendo mención de los códigos en remplazo de sus nombres. Todos estos datos se pueden ver en las tablas 1 y 2 .

Tabla 1. Colaboradores de entrevistas / codificación

\begin{tabular}{|l|l|l|l|l|l|l|}
\hline Nombre & Edad & Profesión & $\begin{array}{l}\text { Tiempo de } \\
\text { estancia en } \\
\text { Cuenca }\end{array}$ & $\begin{array}{l}\text { Motivos de estancia en } \\
\text { Cuenca }\end{array}$ & Origen & Código \\
\hline Mary & 58 & Escritora & 5 años & Salud de su esposo. & $\begin{array}{l}\text { Estados } \\
\text { Unidos }\end{array}$ & E1 \\
\hline Santiago & 42 & Profesor & 3 años & Trabajo en la docencia & España & E2 \\
\hline María & 28 & Violinista & 15 meses & $\begin{array}{l}\text { Búsqueda de trabajo - } \\
\text { trabaja en las calles y bares } \\
\text { tocando el violín }\end{array}$ & Venezuela & E3 \\
\hline Pablo & 33 & Chef & 4 años & $\begin{array}{l}\text { Trabajo - administra su } \\
\text { negocio de alimentos }\end{array}$ & Uruguay & E4 \\
\hline Jhon & 47 & Administrador & 2 años & $\begin{array}{l}\text { Administra su tienda de } \\
\text { joyas }\end{array}$ & Colombia & E5 \\
\hline
\end{tabular}

Elaboración y fuente: la autora

Tabla 2. Auto - etnografía

\begin{tabular}{|l|l|l|l|l|l|l|}
\hline Nombre & Edad & Profesión & $\begin{array}{l}\text { Tiempo de estancia } \\
\text { en Cuenca }\end{array}$ & $\begin{array}{l}\text { Motivos de estancia } \\
\text { en Cuenca }\end{array}$ & Origen & Código \\
\hline $\begin{array}{l}\text { Investi- } \\
\text { gadora }\end{array}$ & 36 & Profesora & 10 meses & Trabajo & Ecuador & AE1 \\
\hline
\end{tabular}

Elaboración y fuente: la autora 
En las tablas se puede ver que el origen de nacimiento de los colaboradores, se sitúa en países como Estados Unidos, Venezuela, Uruguay, España, y Ecuador en el caso de la auto-etnografía. En ese sentido, se hace válido hacer uso del término, comunitas multiculturales, porque estás como asienta Turner (1969) representan "el ahora" de los conjuntos poblacionales en la ciudad de Cuenca. Posteriormente, como lo expresan las tablas 1 y 2 , los motivos de permanencia difieren entre temas la laborales, de salud, o de seguridad. Mientras que sus edades oscilan en una media de 20 y 50 años, sus actividades profesionales, son consideradas diversas, y van desde la docencia en instituciones universitarias, pasando por connotaciones artísticas, intelectuales y de servicios.

El enfoque mixto del trabajo, se complementa con una investigación de tipo documental, apoyada en aportes teóricos de estudios que revisan la realidad social de Cuenca, cuyas aproximaciones alumbran temas neurálgicos como la migración, los nuevos asentamientos poblaciones, la distribución del espacio verde por habitante y las realidades adyacentes como el turismo, la cultura y la educación.

\section{Discusión y Resultados}

\subsection{Las transformaciones sociales de la ciudad de Cuenca.}

La ciudad de Cuenca se ha convertido uno de los destinos más atractivos en Latinoamérica, por las condiciones de vida que ofrece para nacionales y sobre todo extranjeros.

Las denominaciones a las que se ha hecho merecedora la ciudad, en calidad de mejor destino de vacaciones cortas en Latinoamérica, reconocimiento otorgado por el World Travel Award WTA (2018) considerados como los Oscar del Turismo y por la Revista International Living, Ecuador, quien señala refiriéndose a la ciudad que se trata del:

Mejor destino para jubilados extranjeros. Desde hace más de 5 años ha estado en los primeros lugares como el mejor paraíso para jubilarse. Son 8 las categorías rigurosamente evaluadas como: bienes raíces, beneficios especiales para retirados, costo de vida, facilidad de integración, entretenimiento y amenidades, servicios de salud, infraestructura para retirados y clima. En el 2012 la revista International Living clasificó a Cuenca como el primero en el Ranking mundial. (Burbano, 2013, p. 43)

Han propiciado que se convierta en un espacio ciudadano, que, a más de llamar la atención por lo expuesto, ratifica condiciones de vida favorable, que matizan entre lo natural, cultural y lo económico.

Su revisión histórica y social en la última década, la colocan en la mirada global, como territorio de destino en los temas emergentes de movilidad humana, para ciudadanos provenientes de Estados Unidos y Venezuela, muy particularmente. Esas nuevas configuraciones sociales, subrayan en el imaginario colectivo que, "lo global se localiza, de forma socialmente segmentada y espacialmente segregada, mediante los desplazamientos humanos provocados por la destrucción de viejas formas productivas y la creación de nuevos centros de actividad" (Borja, Castells y Benner, 1998, p.1).

A partir de esto y de un acercamiento por medio de las entrevistas, se han podido determinar y comprender diversas posturas y dinámicas sociales, desdibujando desde en un primer plano restricciones y encarecimiento, como principales motivos para elegir a Cuenca, como destino de residencia. De esta manera, lo apuntan los entrevistados:

"Nuestra pensión de jubilados en Estados Unidos si podría alcanzarnos para vivir, pero no podríamos ahorrar, y no queremos vivir con lo justo. Tenemos el presentimiento que cualquier momento la vida será más cara y no nos permitirá vivir" E1. 
"Los colegas en Ecuador, me decían que la economía no era buena, porque la clase trabajadora gana muy poco para el costo de vida, pero socialmente hay menos problemas, en algunas ciudades se puede vivir con tranquilidad y hay ciudades que están cerca del mar o de las montañas, en España eso es más difícil" E2.

De su lado, los entrevistados han indagado sobre la misma pregunta a la entrevistadorainvestigadora, quien, para establecer el acercamiento previo con los colaboradores, ha manifestado que es ecuatoriana, pero no ha nacido en Cuenca. En virtud de eso, tres de los cuatro informantes, han revertido la pregunta hacia un: ¿Y tú?

"Mis motivos son laborales, aunque Cuenca siempre llamó mi atención como destino para pasear, no me había planteado antes vivir aquí, me siento muy bien, tengo la sensación de vivir en el extranjero, es una ciudad con pulso aún calmado, puedo ir a trabajar caminando, pasear y sentirme segura" AE1.

Llama la atención que, en adelante se puede percibir que esos motivos de desplazamiento, están encausados en casos puntuales, en base a decisiones de último momento, sorteando la suerte y la información boca a boca, de coterráneos de los colaboradores.

"Tuvimos que salir de Venezuela por la crisis, viajamos con unos primos y cuando llegamos a Quito en bus, no sabíamos si quedarnos o irnos a Guayaquil. Entonces nos dijeron otros venezolanos, que Cuenca era mejor lugar para ir" E3

"Estuvimos en Quito con mi pareja dos meses, pero unos amigos nos comentaron que en Cuenca el negocio estaba mejor y era más seguro para vivir, y es cierto" E5

Ya en territorio cuencano, luego de conocer las razones que hoy en día los sitúan en el territorio, todos han coincidido que, si bien para algunos ha sido difícil la adaptación, el "ecotono" en el que se convierte la ciudad, al ser concebido como "una zona de transición natural entre dos ecosistemas distintos" (Carbonell y Torrealba, 2007, p. 230), mitiga cualquier tipo de segregación socio cultural que pudiera existir.

"Ver gente de todas partes del mundo, te da confianza. En los parques niños jugando, la gente echada sobre el pasto" E4

"Siento que la ciudad no es ni muy pequeña ni muy extensa. Tiene las dimensiones para mi perfectas para comprar comida, ir a los hospitales, los ríos son muy bonitos y cercanos" E1

"Me siento cómoda, aunque echo de menos a mi familia. Que haya más venezolanos, que podamos vernos en las calles, en los parques, es muy bueno, nos hace sentir en casa" E3

Estas respuestas preliminares y parte de la auto-etnografía, brindan las pautas para describir cómo se concibe la ciudad en términos de acercamientos, de cohesión social. Es así que, en ese proceso de auto-reflexión, se afirma:

"Al llegar a Cuenca, lo primero que pedí, fue la dirección de la Universidad donde iba a laborar. Así, previo al inicio de labores, miré el mapa para ubicar la dirección (avenida 12 de abril y Loja), pero sobre todo hacerme del lugar, ubicarme, en relación con los lugares que serían importantes para mi desempeño. Un lugar para vivir, centros de abastecimiento de alimentos, centros médicos, zonas seguras para caminar, lugares que me permitieran vivir en una ciudad de forma tranquila.

Recuerdo que el primer día de funciones, la arrendataria de casa, me dijo que podía bajar caminando hacia la Universidad. De tal forma, que empezó mi andar por Cuenca. Si bien no era la primera vez que vivía fuera de casa, era consciente que cada situación y cada contexto, sería diferente, de tal forma que pueden llegar a hacer sentirnos llenos o vacíos.

Una de las primeras cosas que descubrí, fue llegar al final de la Calle Larga, y observar otro ambiente ciudadano. Unas escalinatas y un río, separaban imaginariamente la ciudad de la universidad, o más bien las unían. El río Tomebamba, se convierte en ese túnel natural, que por 
un lado dejaba atrás, la bruma citadina, siendo capaz de vaciar o disipar el ruido, con el trinar de los pájaros, con el olor a bosque y con el ruido del agua recorriendo las piedras del rio. Al cruzar el rio, la sensación de relajación es otra, ese sentido metafórico de corredor natural, no está tan alejado de la realidad, pues cruzar la frontera del río, equivale a dotarse de una dosis de aire puro y de carga emocional". AE1

De esto, algunos estudios sostienen la importancia de establecer vínculos entre la ciudad y elementos culturales o naturales, como fines para mejorar la calidad de vida, (Solano,1983; Santos, 2001; Castells, 1997; Margulis, 2002; Canclini, 1997), reafirmando esa dinámica activa, provocada por los movimientos migratorios y como efectos de los cambios globalizantes.

Por otro lado, se recrean también otras razones, recogidas en la investigación de Burbano (2013), cuyos sujetos de estudio son jubilados que viven en Cuenca. Ellos desde una postura individual, plantean cuales son las principales motivaciones para elegir a la ciudad como destino de residencia, una vez que el autor les ha preguntado: ¿Que lo motivó a escoger Cuenca Ecuador?

"Cuando llegué me pareció un lugar muy acogedor y con un toque de europeo lo cual me fascino. El costo de vida me deslumbró y su tranquilidad fue lo que más me motivó" (Burbano,2013, pp. 74-75)

"Me encantan muchas cosas, pero lo mejor es la falta de presión. Es una vida muy relajada y en todo lugar te reciben muy bien. Creo que lo mejor es las personas que son muy acogedoras" (Burbano,2013, p. 78)

"Es lo suficientemente grande para encontrar todo lo que mi cultura americana desea tener. Es hermoso. ¡El clima juega un rol muy importante para mí! Me encanta que no sea muy frio ni muy caliente". (Burbano,2013, p. 80)

Estas respuestas coincidentemente se alinean a las obtenidas de los colaboradores de la investigación, dando pautas para percibir que estos espacios ciudadanos, no planificados y producto de las transformaciones sociales, permiten asimilar nuevos enfoques de hacer ciudadanía, de interrelacionarse y de reproducir paralelamente otros entornos.

En tal sentido, las familias, las niñas y niños, jóvenes y adultos, encuentran ambientes de esparcimiento y participación global en parques, plazas, mercados o en sus calles, de manera muy significativa.

Las áreas verdes en Cuenca, simbolizadas en parques, riberas de los ríos, lagunas y montañas cercanas, "por un lado contribuyen en el aspecto social, siendo espacios públicos, de recreación, ocio y democratizadores, además de ser un importante factor en el ámbito ecológico" (Sierra y Ramírez, 2010, p.6), lo que constituye en una de las razones más, por las que aquellos que conocen la ciudad, optan por asentarse indefinidamente. Tienen una incidencia también predominante, las calles que proyectan un legado histórico y determinante en la cultura local. Hoy por hoy, contrastan con coloridos murales, que no sólo evocan la voz de la opresión, sino también la fusión de viejas y noveles miradas. La ciudad se empieza a leer en el arte, en la música, en las expresiones culturales diversas, que son capaces de dejarse sentir en el ciudadano de a pie y de transporte, que cada vez más asimila una nueva forma de vida,

En el caso específico de Cuenca, las personas ya no se sorprenden tanto al ver a ciudadanos de diferentes nacionalidades en sus calles o compartiendo el transporte urbano público con ellos. Sin embargo, los cuencanos nos hemos caracterizado, en su mayoría, por ser conservadores en nuestra manera de pensar, dicho aspecto puede resultar chocante para el extranjero. Aunque algunos se han adaptado a la ciudad, existen otros que quieren que la ciudad se adopte a ellos. Ambas partes podrían optar por una construcción mutua. (Romero,2018, p.50)

Esa "progresiva concienciación sobre la diversidad real existente en la sociedad, que refleja no solamente la dimensión de género, etnia y origen socioeconómico, sino también otros aspectos (estilos de aprendizaje, ritmos, intereses, inteligencias diversas, etc.)" (Rego, 2010, 
p.86) se empieza a enraizar en una ciudad de corte global, que ha sido capaz de adaptarse a los cambios propuestos por la modernidad, sin perder la esencia histórica que recorre sus pasadizos coloniales.

\subsection{La academia como eje dinamizador de las sociedades interculturales}

Cuenca, la ciudad que cuenta con una población de 505.585 según datos del Censo 2010 del INEC, se ha convertido en los últimos años en una de las principales sociedades multiculturales de la región, donde además del análisis de las complejas relaciones sociales, se contempla lo social "no sólo en términos de cultura, sino también en términos de etnicidad, lengua, denominación religiosa y/o nacionalidad" (Dietz, 2017, p.192). Su dinámica obedece a una herencia cultural, marcada por asimetrías sociales, políticas y económicas que, a causa de los movimientos y transformaciones sociales, han dado paso a una articulación global, que busca "formar ciudadanos interculturales" (Johnson, 2015). Esta ciudad con miras a fundarse en una sociedad intercultural, representa en la actualidad el puerto seguro de cientos de personas que, además de un hogar buscan condiciones óptimas de salud, seguridad y educación.

De esta última, es posible determinar su trascendencia en el desarrollo de la ciudad, no sólo por el número de instituciones educativas públicas y privadas, por el carácter formativo y cultural que se expresa de forma espontánea en sus arterias, sino también por el vaivén de estudiantes transitando, que no hacen más que dejar notar a una población universitaria que ha convertido a Cuenca, en un espacio de estudio, movilización y cohesión social. Invitando, por tanto, a recordar que esas "estructuras en las geopolíticas del saber solo pueden ser cambiadas tras una aceptación social y la co-existencia de las culturas presentes" (Ströbele-Gregor. Kaltmeier y Giebeler, 2011, p. 7) a través de estas activas poblaciones estudiantiles.

Frente a estos hechos, nos situamos ante un entorno que paulatinamente ha dado paso a consolidarse como una urbe académica, conjugando lo educativo, social, cultural y moderno en sus estrechas calles, en aras de ser percibida como una ciudad universitaria.

Tabla 3. Instituciones de Educación Superior (IES) en Cuenca

\begin{tabular}{|l|l|l|l|}
\hline Institución de educación superior & Tipo de institución & Años de funcionamiento & Ubicación \\
\hline Universidad de Cuenca & Pública & 151 años & Zona urbana \\
\hline Universidad del Azuay & Privada & 50 años & Zona urbana \\
\hline Universidad Politécnica Salesiana & Privada & 24 años & Zona urbana \\
\hline Universidad Católica de Cuenca & Privada & 38 años & Zona urbana \\
\hline Universidad Particular de Loja & Privada & 30 años & Zona urbana \\
\hline
\end{tabular}

Elaboración y fuente: propia del autor con datos tomados de los sitios web de las IES.

Las ciudades universitarias en el mundo, se han conformado en base a las poblaciones estudiantiles que habitan sus entornos. En los últimos años, la ciudad se va trasladando a las universidades, o se van fundado a su alrededor, bajo el nombre de sociedades del conocimiento. En Ecuador tenemos el caso de Yachay, en otras latitudes muchos y muy variados ejemplos, entre los que se mencionan, la -Cidade Universitaria- en Lisboa, el -Barcelona Knowledge Campus- en España o la universitas professorum ac studiosorum en Lovaina - Bélgica. Cuyos propósitos acordes a, Del Valle (2009), se articulan en función de:

Promover su desarrollo mediante el reforzamiento de su dotación en recursos de conocimiento, la capacidad creativa de su población y sus instituciones, la existencia de redes de cooperación 
entre ellos y la generación de procesos de aprendizaje colectivo, que potencien la aparición de procesos de innovación económica y social (p.119).

Y en las premisas para facilitar la vida de los estudiantes con servicios de calidad, que además del componente educativo, activan otros dispositivos como salud, deporte, diversión y alimentación, logrando de otra mano, involucrarlos como parte del "vecindario residente habitual" (Hernández, 2004, p.138).

De alguna manera, esta realidad no dista de lo que viene ocurriendo en Cuenca, pues uno de esos axiomas culturales, se ha forjado desde hace 150 años en la ciudad, con el ejemplo de la Universidad de Cuenca, o estatal como es conocida localmente. Su institucionalización tiene unas particularidades multi e interculturales, que no sólo recaen en lo académico, al constituirse en un centro de formación accesible a la población, con una oferta educativa, que a través de los años ha ido considerando las necesidades de sus habitantes en relación directa con el paisaje y con los ejes de producción, sino también que trasciende y cohesiona su espíritu con la ciudad, proponiendo una fragmentación cultural, urbana y rural.

Vale resaltar que el centro histórico y comercial de la ciudad, está a pocos pasos del campus central (avenida 12 de abril). Lo cual significa que llegar al centro de estudios, involucra dar unos pasos en medio de un remanso de bosque, que tiene el río como principal panorama, recreando ese pasillo natural que se describía en la auto etnografía. "pocos sitios que he visitado tienen esa característica natural" AE1.

Estos espacios previos al ingreso al campus, se entreveran como sitios de convivencia, con la capacidad de servir de aula abierta en un entorno dual. "En ocasiones he podido ver a estudiantes de arquitectura, sentados en el césped alrededor del rio, pintando el paisaje natural o las edificaciones que están alrededor" E2.

Ocurren situaciones similares, en el campus de la Universidad del Azuay, que está ubicado sobre las riberas del río Yanuncay y cuyo paisaje es recurrente, en una urbe como Cuenca rodeada por cuatro ríos (Tarqui, Yanuncay, Tomebamba y Machángara).

"Hay mucha vida y movimiento alrededor de las universidades, están los ríos y gente yendo a la universidad o haciendo deporte" E4.

Todas estas instituciones de educación superior, están ubicadas en el marco urbano o relativamente cerca del centro de la urbe. $Y$ han llegado a descentralizar varias de sus unidades o facultades, desde un pensamiento democratizador, que permite distribuir sus campus en sitios estratégicos de la ciudad o alrededor de ella. De esta forma, no llama la atención que, en el centro histórico, la zona moderna o en las periferias, se puedan encontrar facultades o centros adscritos. Que más allá de desconcentrar las masas estudiantiles en un solo centro universitario, permiten desplegar ese sentir académico en diferentes dimensiones.

A pesar que "el gran tema de los nuevos movimientos sociales es el problema de la identidad socio cultural" (Dietz, 2012, p.17), son estos imaginarios socio formativos, donde se logran estructurar y construir espacios para el diálogo y la convivencia, de una manera espontánea, teniendo a la academia como aliada. Es así, que la interculturalidad se asienta en un terreno democrático y con mayor frecuencia se puede observar a estudiantes haciendo uso de esos ambientes, dentro o fuera, alrededor o en los parques de la ciudad, con fines educativos, recreativos, culturales. Llevando a convertirlo en sitios donde convergen familias, amigos y la alteridad en un magno y aprovechador sentido. Para lograrlo, se ha hecho necesario, repensar la sociedad en base a esos cambios y transformaciones sociales, que en vez de resistir se adaptan y sobreviven al cambio de manera positiva, propiciando lugares donde se cohesionan la diversidad cultural, étnica, religiosa y de género.

Esto ha advertido a la academia, en los últimos años ha repensar y rediseñar los modelos pedagógicos y las prácticas educativas, en función de un acercamiento a la ciudad y su sociedad. Enmarcándose en la adquisición y el desarrollo pleno de las competencias interculturales, basadas en "la producción de conductas pro-sociales, de participación, aceptación y solidaridad 
básicas, constituyen objetivos de un modelo pedagógico de aprendizaje cooperativo. (Rego, 2010, p.95).

Las universidades, las escuelas y las instituciones educativas actualmente, reflejan un "sistema educativo que afronta hoy el reto de ofrecer la mejor educación, desde el respeto y el reconocimiento, a los que tienen por lengua materna la del lugar y a los que no" (Enguita, 2001, p.7) transversalizando los saberes integradores en sus ofertas curriculares. Pues resulta necesario, que las instituciones de orden académico y social, continúen proyectando y potenciando a la urbe, como un núcleo garantista para alcanzar no únicamente una educación de calidad, sino una ciudadanía participativa y global, que usa la diferencia para enriquecer y fortalecer las complejas relaciones sociales.

\subsection{La sociedad intercultural y los desafíos poblacionales}

Vale recordar que, "cuando los discursos multiculturalistas comienzan a migrar de un contexto a otro, sus puntos de partida originales (...) suelen terminar mezclados, confundidos y supuestamente neutralizados en su poder para moldear las "soluciones" educativas en el nuevo contexto. (Dietz, 2017, p.196).

En ese aspecto, la globalización, el progreso tecnológico y la modernidad, ponen en debate un diálogo que cuestiona, replantea y re piensa, si esas nociones elementales, que proponen a la interculturalidad como solución para mitigar los problemas que aquejan a las sociedades multiculturales, son un punto de llegada en la construcción de las urbes contemporáneas. O si bien, se estructuran como "algo por construir", Walsh (2009), en constante transformación, sin recetas prefijadas. Puesto que "esas manifestaciones de la aceleración contemporánea carecen de poder analítico". (Santos y Silveira, 2018, p.102), y corren el riesgo de recaer en sincretismos sociales o en la esencialización de una cultura, que resiste a los alcances de la modernidad. Para esto únicamente, "no es suficiente reconocer la diferencia que se está dando en los sistemas multiculturales" (Ströbele-Gregor et al., 2011, p.7), sino siendo conscientes de ella y de sus problemáticas realidades. Por un lado, hay quienes consideran que "la violencia y la inseguridad pública, la inabarcabilidad de la ciudad llevan a buscar en la intimidad doméstica, en los encuentros confiables, formas selectivas de socialibilidad" (Canclini, 2012, p. 266), que trae consigo la globalización, alteran las estructuras sociales negativamente. A esto se suman los mensajes continuos que surgen de los múltiples canales de comunicación, que no dejan de hacer que "Ias peripecias sociales se reciban en la casa, se comenten en familia o con amigos cercanos" (Canclini, 2012, p. 268).

Por otro lado, también se cuestiona el crecimiento demográfico que generan esos nuevos asentamientos, en función de "las pocas posibilidades que ofrece el espacio público verde a escala de barrios y manzanas de la ciudad, su ausencia no potencia la interacción social y el derecho al uso de los espacios públicos urbanos". (Cordero et al., 2015, p.128).

Para el fin, es importante incorporar la visión que propone a "la interculturalidad como recurso funcional para mejorar las relaciones sociales (...) mediante la identificación de características individuales (falta de competencias, falta de habilidades de comunicación, falta de capital humano, etcétera) como carencias y causas de exclusión, de discriminación y de relaciones asimétricas persistentes" (Dietz, 2017, p.194). Intersectados por otros "abordajes describen, especialmente, el papel de la economía mundial - la competitividad, el mercado mundial, entre otros - y el papel de la nueva cultura - los signos y símbolos globales -, aunque ignorando la resistencia de los lugares y su proceso de singularización”. (Santos y Silveira, 2018, p.105).

En esta revisión, hemos podido notar que la perspectiva intercultural, en algunas sociedades "se utiliza para referir a la diversidad provocada por la migración, mientras que en otras la misma noción se aplica para las interacciones entre pueblos indígenas y descendientes de colonizadores" (Dietz, 2017, p. 192), en ambos casos se contraponen a los ideales sociales y culturales, de una población ciudadana, que recurre a la nostalgia humana, para re fundar, 
esos "no lugares" Auge (1992), que la globalización ha mutado. La incorporación de estos no lugares, construidos en el imaginario colectivo, se estructuran en función de "bases materiales que se localizan diferencialmente en el territorio, al tiempo que la realidad del lugar está dada por el orden espacial y temporal del conjunto de objetos. (Santos y Silveira, 2018, p.106), desplegándose "llenos de significados y valores, que son inseparables de la experiencia de quienes lo habitan, de sus pensamientos y sentimientos» (García Ballesteros, 1992, p.11).

Finalmente se plantea la desapropiación de un punto de inicio, que no condiciona el destino de un enfoque, sino más bien encuentra en la transformación permanente, las formas de subsistencia de las sociedades contemporáneas. Esta propuesta para mirar la ciudad y la sociedad en conjunto, subraya que "hacer cultural studies no significa transitar sendas que alguien fijó de antemano" (Hall y Mellino, 2011, p. 14), significa también cuestionar las disciplinas, las tradiciones educativas y reinventar el sentido social haciendo uso del espacio natural y cultural, para potenciar mejores relaciones y nuevas formas de convivencia entre todos.

\section{Conclusiones}

Precisamos entonces, una academia con una visión más amplia de lo que significa educar sin normar, sin hacer uso de un poder hegemónico para acentuar las diferencias y menos para reafirmar minorías. Pues los aportes de aquellas mal llamadas minorías, están siendo significativos en las miradas transformadoras que la academia está agregando a sus curriculums, "desde campos como la filosofía, la demografía, la sociología, la política, la informática o la economía se está propiciando la creación de una sociedad más interrelacionada y multicultural" (Rego, 2010, p.87).

Las ciencias, artes y tecnologías están cambiando el rumbo de la educación en el mundo. Cada vez más nos basamos en modelos pedagógicos que entrelazan el conocimiento universal, con el que nace desde la "ecología de saberes" (De Sousa Santos 2014), propia de nuestros pueblos indígenas, montubios, afroecuatorianos, de todas aquellas culturas hibridas que se vienen formando través de la modernidad como un plus de desarrollo. Nos convoca un momento para pensar y repensar otros tipos de instituciones, con cambios estructurales físicos y socio culturales, donde tenga cabida un pensamiento plural que conciba a los movimientos sociales, "como realidades colectivas, socialmente construidas" (Melucci, 1995, p.110). Teniendo de base las nociones de los estudios que ofrece la antropología, la etnografía, como cuerpos de resistencia que insisten positivamente en mostrar una realidad más cercana. Esa visión contemporánea que nos ofrece después de los años 80 , cambia esa percepción estática de la cultura, hacia una noción más dinámica, relacionada con prácticas de interacción, construidas permanentemente en la espontaneidad y la realidad del contexto. Es entonces que se espera que, la interculturalidad encuentre su realización en la praxis, teniendo presente que "en las sociedades multiculturales hay conflictos, incluso graves conflictos, nadie puede negarlo". (Rego, 2010, p.92) pero que sin embargo se puedan mediar para mejorar las situaciones y relaciones, en medio de un ambiente de participación ciudadana que fomenta la convivencia armónica.

Frente a esto, potenciar esas relaciones más incluyentes, no sólo requiere cambiar las estructuras actuales, sino también conocer cómo se han estructurado en el pasado, como se articulan en el presente y cuál es el panorama que se avizora en el futuro, en miras de relaciones basadas en la justicia social y en una justicia cognitiva.

Es imprescindible dar paso al desarrollo de habilidades, que acuñan el bagaje cultural multidisciplinar de todas las personas. Pues la interculturalidad compete a todos, quienes somos parte de una especie humana hiperdiversa, que busca acciones colectivas que prioricen los principios de igualdad y justicia social, donde cada uno puede tener acceso a oportunidades que mejoren sus condiciones de vida.

Para ello, seremos cada vez más conscientes que, esto no se puede lograr sin el acompañamiento de la academia, la sociedad civil, instituciones públicas y privadas y el estado. 


\section{Referencias bibliográficas}

Abdallah Pretceille, M., y Prat, F. (2001). La educación intercultural. Barcelona, España: Idea Books.

Auge, M. (1992). Non-Lieux. Introduction à une anthropologie de la surmodemité. Paris, Francia: Éditions du Seuil.

Asamblea, D. E. (2009). Plan Nacional del buen Vivir. Quito, Ecuador: Senplades.

Bianco, C., Lugones, G., Peirano, F., y Salazar, M. (2016). Indicadores de la Sociedad del Conocimiento: aspectos conceptuales y metodológicos. Bogotá, Colombia: Colciencias.

Blanco, M. (2012). Autoetnografía: una forma narrativa de generación de conocimientos. Andamios, 9 (19), 49-74.

Borja, J., Castells, M., Belil, M., y Benner, C. (1998). Local y global: la gestión de las ciudades en la era de la información. Madrid, España: Taurus.

Burbano, C. (2013). La inmigracion de jubilados extranjeros hacia latinoamerica: un modelo en cuenca: ecuador (tesis de grado) Universidad de Especialidades Espíritu Santo UEES, Guayaquil, Ecuador.

Canclini, N. (2012). Culturas híbridas. México DF, México: Debolsillo.

Carbonell, F., y Torrealba, I. (2007). Conservación en ecotonos interculturales y transfronterizos: Una visión integral en la Reserva de Biosfera La Amistad, Costa Rica-Panamá. Textual, análisis del medio rural latinoamericano, Revista de la Universidad Autónoma de Chapingo, 50, 217-242.

Castells, M., y Romero de Solís, I. (1997). Movimientos sociales urbanos. España: Siglo XXI de España editores.

Coulby, D. (2006). Intercultural education: theory and practice. Intercultural education, 17(3), 245-257.

Constituyente, E. A. (2008). Constitución de la República del Ecuador.

Cordero, P., Vanegas, S., y Hermida, M. (2015). La biodiversidad urbana como síntoma de una ciudad sostenible. Estudio de la zona del Yanuncay en Cuenca, Ecuador. Universidad de Cuenca.

De Solano, F. (1983). Estudios sobre la ciudad iberoamericana. Editorial CSIC-CSIC Press.

Del Valle, R., Moral, S., Aragón, A., y Balestena, G. (2009). Sistema urbano y sociedad del conocimiento: hacia una tipología de las ciudades españolas. Investigaciones regionales: Journal of Regional Research, (16), 117-142.

Dietz, G. (2012). Multiculturalismo, interculturalidad y diversidad en educación: una aproximación antropológica. México DF, México: Fondo de cultura económica.

Dietz, G. (2017). Interculturalidad: una aproximación antropológica. Perfiles educativos, 39(156), 192-207.

Eckholt, M. (2018). Espacios de paz. Nuevos caminos de teologías interculturales de la paz. Teología, 53(119), 115-127.

Ecuador, G. N. (s/f). Yachay, ciudad del conocimiento.

Enguita, M. (2001). La educación intercultural en la sociedad multicultural. Organización y gestión educativa, 6, 3-9.

Ellis, C., y Berger, L. (2002). Their Story/My Story. Our Story: Including the Researcher's 
Experience in Interview Research. (Ed.), Inside Interviewing (pp. 467-482). California, Estados Unidos: Sage Publications.

Frutos, A. (2015). Comunidades interculturales y democráticas: un trabajo colaborativo para una sociedad inclusiva (133). Narcea Ediciones.

García, A. (1992). Las aportaciones de la Geografía Humanística». In García Ballesteros A. (éd.): Geografía y Humanismo. Barcelona, España: Oikos-Tau.

Galeano, E. (2014). Ser sentipensante. Recuperado de: https://www. youtube. com/watch.

Hall y Mellino (2011). Cultura y Poder. Buenos Aires, Argentina: Amorrortu editores.

Hernández, M. (2004). Ciudades universitarias y orden público en la Edad Moderna. Cuadernos de Historia Moderna. Anejos, 3, 137-162.

Johnson, D. (2015). Formar ciudadanos interculturales en un mundo global: algunas notas desde los estudios curriculares. Diálogo andino, (47), 7-14.

Marcovitch, J. (2002). La universidad (im) posible. 2. Madrid, España: Ediciones AKAL.

Margulis, M. (2002). La ciudad y sus signos. Estudios sociológicos, 515-536.

Mato, D. (2009). Instituciones Interculturales de Educación Superior en América Latina. Procesos de construcción, logros, innovaciones y desafíos. UNESCO. IESALC.

Marcuse, H. (1968). El Final de la Utopía. B945. M37 1981.

Melucci, A. (1995). The process of collective identity. Social movements and culture, 4, 41-63.

Montero-Sieburth, M. (2006). La auto etnografía como una estrategia para la transformación de la homogeneidad a favor de la diversidad individual en la escuela. In Universidad de Massachussets-Boston. Recuperado de http://www. uned. es/congreso-inter-educacionintercultural/Grupo_discusion_1/74. pdf.

Odina, T., y Del Olmo, M. (2009). Educación intercultural: perspectivas y propuestas. Madrid. España: Editorial Universitaria Ramón Areces.

Reglamento General, A. La Ley Orgánica De Educación Intercultural (Ecuador). (s/f). Registro Oficial Suplemento, 754.

Rego, M. (2010). La ciudadanía y la interculturalidad en la sociedad del aprendizaje. Edetania: estudios y propuestas socio-educativas, (37), 83-107.

Romero, M. (2018). Comunicación Intercultural. Estudio del caso: presencia de extranjeros en la ciudad de Cuenca (tesis de grado). Universidad de Cuenca, Cuenca, Ecuador.

Roca, E. (2011). Campus y ciudad: la experiencia del Barcelona Knowledge Campus. Revista Iberoamericana de Urbanismo, (5), 95-105.

Santos, M., y Silveira, M. (2018). Más allá de las metáforas... Una geografía de la globalización. Estudios geográficos, 59(230), 99-112.

Ströbele-Gregor, J; Kaltmeier, O, y Giebeler, C. (2010). Construyendo Interculturalidad: Pueblos Indígenas, Educación y Políticas de Identidad en América Latina. Frankfurt, Alemania: Druckreif Gründenseestr.

Sierra, I, y Ramírez-Silva, J. (2010). Los parques como elementos de sustentabilidad de las ciudades. Revista Fuente, 2(5), pp. 6-14.

Santos, B. D. S. (2001). Los nuevos movimientos sociales. OSAL: Observatorio Social de América Latina, (5), 177-188. 
Santos, B. (2014). Más allá del pensamiento abismal: de las líneas globales a una ecología de saberes. Epistemologías del Sur (Perspectivas), 21-66.

Schensul, J., y Le Compte, M. (Eds.). (1999). Ethnographer's toolkit. Nueva York, Estados Unidos: NC State University.

Tum, R. M. (2002). El sueño de una sociedad intercultural. Cinco ciudadanías para una nueva educación. 63-82

Turner, V. (1969). Liminality and communitas. The ritual process: Structure and anti-structure, $94,130$.

Walsh, C. (2009). Interculturalidad, estado, sociedad: luchas (de) coloniales de nuestra época. Quitor, Ecuador: Universidad Andina Simón Bolívar.

World Travel Awards. (2018). Recuperado de https://www.worldtravelawards.com/winners/2018/ south-america

Zapata-Barrero, R. (2015). Las condiciones de la interculturalidad. Gestión local de la diversidad en España. Valencia, España: Tirant Lo Blanc. 